\title{
Anti-Ro antibody and clinical manifestations: lessons from systemic lupus erythematosus in the elderly
}

\author{
Rui-Xue Leng • Wei-Zi Qin • Hai-Feng Pan • \\ Dong-Qing Ye
}

Received: 31 May 2010 / Accepted: 2 June 2010 / Published online: 12 June 2010

(C) Clinical Rheumatology 2010

\section{Dear Editor,}

As we know, previous studies have shown the association of systemic lupus erythematosus (SLE) and anti-Ro antibody with a series of clinical manifestations. However, available evidence suggests that there still exist a large number of inconsistencies. Recently, Paz et al. reported that there was no direct relationship between anti-Ro antibody and cutaneous photosensitivity in SLE patients [1]. Another study also found that anti-Ro antibody was not associated to photosensitivity. However, there was an association with anti-La antibody. Moreover, in multivariate analysis, patients with anti-Ro antibody has 1.63 (95\% CI, 1.07-2.50) more risk to develop cutaneous vasculitis than patients without this antibody [2]. Interestingly, Shinjo et al. showed that SLE cutaneous vasculitis group was not associated to anti-Ro antibody, but the group had a higher frequency of photosensitivity. Additionally, the authors also found that had a higher frequency of Raynaud phenomenon in cutaneous vasculitis group [3].

Clinical manifestation in late onset SLE may provide a good reference for this disagreement. Rovenský et al. systematically reviewed clinical manifestation for SLE in the elderly and found that late onset SLE patients

Rui-Xue Leng and Wei-Zi Qin contributed equally to this work and should be considered co-first authors.

R.-X. Leng • W.-Z. Qin · H.-F. Pan · D.-Q. Ye $(\bowtie)$

Department of Epidemiology and Biostatistics,

School of Public Health, Anhui Medical University,

81 Meishan Road, Hefei 230032 Anhui,

People's Republic of China

e-mail: ydq@ahmu.edu.cn manifested higher rate of positive findings of anti-Ro and anti-La antibodies. In contrast, skin vasculitis, photosensitivity and Raynaud phenomenon occured rarely in the elderly patients [4]. The review may prove that anti-Ro antibody is associated to prevalence of anti-La antibody but not photosensitivity, cutaneous vasculitis and Raynaud phenomenon, which is in agreement with most findings from the reports discussed above [1-3]. It should be noted that further studies are required to clarify precise molecular mechanism. In spite of this, clinical manifestation in late onset will provide a better understanding for the role of anti-Ro antibody in SLE.

Disclosures None

\section{References}

1. Paz ML, González Maglio DH, Pino M, Ferrari A, Weill FS, Nasswetter G, Leoni J (2010) Anti-ribonucleoproteins autoantibodies in patients with systemic autoimmune diseases. Relation with cutaneous photosensitivity. Clin Rheumatol. doi:10.1007/ s10067-010-1481-8

2. Fukuda MV, Lo SC, de Almeida CS, Shinjo SK (2009) Anti-Ro antibody and cutaneous vasculitis in systemic lupus erythematosus. Clin Rheumatol 28:301-304

3. Shinjo SK, Bonfá E (2010) Cutaneous vasculitis in systemic lupus erythematosus: association with anti-ribosomal $\mathrm{P}$ protein antibody and Raynaud phenomenon. Clin Rheumatol. doi:10.1007/s10067010-1432-4

4. Rovenský J, Tuchynová A (2008) Systemic lupus erythematosus in the elderly. Autoimmun Rev 7:235-239 